\title{
Numerical investigation of capillary driven droplet breakup due to fluid dynamic instabilities
}

\author{
Patrick Giefer*1, Udo Fritsching ${ }^{1,2}$ \\ ${ }^{1}$ Leibniz Institute for Materials Engineering-IWT, Particles and Process Engineering, 28359 \\ Bremen, Germany \\ ${ }^{2}$ University of Bremen, Particles and Process Engineering, 28359 Bremen, Germany \\ ${ }^{*}$ Corresponding author email: p.giefer@iwt.uni-bremen.de
}

\begin{abstract}
The membrane emulsification process obtains the advantage to formulate liquid/liquid systems in a low shear stress process. Whereas the emulsification process and the influence of the membrane structure and geometry on the final product are well investigated, the liquid deformation and liquid dispersion process inside the membrane structure are quite unknown. In the present study, the droplet breakup mechanism in the vicinity of a solid wall is numerically investigated with OpenFoam using the Volume of Fluid Method. Different wetting conditions on the membrane wall have been implemented. The results show a relation between wetting properties and fluid dynamic disturbances' resp. instabilities that may grow to larger instabilities, resulting in droplet breakup inside the membrane. These results can be used to find the sweet spot of capillary driven droplet breakup with minimum shear force to handle shear sensitive media while keeping a defined mono disperse emulsion.
\end{abstract}

\section{Keywords}

Membrane Emulsification, Droplet Breakup, Fluid Dynamic Instabilities, Wetting

\section{Introduction}

Emulsions as liquid/liquid formulations are widely used in the industry. They play an important role in pharmaceutical- food- and cosmetic applications. The premix membrane emulsification is a low shear stress process which is relevant for handling shear sensitive media, for instance in biological systems. It was first introduced by Suzuki et al. [1] and obtains the advantage of adjusting the final droplet size with an narrow size distribution. This is a key parameter for a tailored high-quality emulsion.

In several studies the influence of process parameters like transmembrane pressure [2], dispersed and continuous phase velocity [3], membrane porosity, and pore size [4], [5] were investigated. On the other hand, the influence of fluid properties like dispersed phase fraction, dispersed and continuous phase viscosity's as well as surfactant concentrations were quantified by several authors [6], [3], [5], [7]. In particular, the influence of surfactants on the final emulsion is widely discussed but not fully understood. The surfactant adsorbs at the liquid/liquid interface, reduce the interfacial tension, and therefore stabilizes droplets from further coalescence [8]. The influence on the droplet breakup is explained by lowering the minimum emulsification pressure for membrane applications. [9] For purely shear driven emulsification processes, it is directly connected with lowering the critical capillary number [10]. Previous studies on idealized membrane pore geometries [11] as well as fully resolved membranes pointed out that the highest shear stresses occur for a short time at the wall, while lower shear stresses occur at the liquid/liquid interface and their absolute value is higher [11]. Schroën et al. [12] pointed out the importance of wettability and surfactant interactions with interfaces. They concluded, that most surface interactions increase the contact angle towards $90^{\circ}$, either through surfactant or the oil that is used.

Although it seems that the emulsification process is deeply understood, the droplet breakup mechanism is still quite unknown, especially under the consideration of membrane wettability. 
The question that needs to be addressed is where does the droplet break up, and what causes the breakup? For cross flow emulsification, work by Peng et al. [13] and Abrahamse et al. [14] leads to a further understanding of the underlying fluid dynamics. For membrane emulsification, van der Zwan et al. [15] divided the breakup mechanism into three categories: (i) Snap-off due to localized shear forces, (ii) Break-up due to interfacial tension effects (Rayleigh and Laplace instability) and (iii) Break-up due to steric hindrance between droplets. A key part is the predeformation of the droplet into a dumbbell shape while entering the pore. It is discussed that this destabilization leads to the minimization of the minimum shear stress necessary for breakup. This is the connecting point between previous studies and the present study. This work focuses on a single droplet breakup in a single membrane pore under the consideration of membrane wettability. Due to the limitation on this system, the category (iii) suggested by van der Zwan [15] will not be taken into account. The main objective is the understanding of the droplet breakup caused by fluid dynamic instabilities. Therefore, computational fluid dynamics (CFD) is utilized.

\section{Materials and methods}

Membranes used in premix emulsification have in general a complex pore structure with a branched network in which break up events occur simultaneously. This fact makes it quite challenging to observe single droplet breakup mechanisms. For this reason, the problem will be abstracted into a cylindrical single pore in which a single droplet emerges as shown in figure 1. The three-dimensional numerical domain is determined by two connected parts, one to initialize the spherical droplet with a length of $2 \mathrm{~mm}$ and the other one is representing the actual membrane pore with a length of $6 \mathrm{~mm}$. The constant velocity is initialized at the left boundary. Constant pressure boundary condition is imposed at the right wall and no-slip boundary condition is imposed at the side walls. The open source software OpenFOAM is used with the multiphaselnterfoam solver. The wettability is implemented with a constant contact angle model. The hexahedral mesh was generated with snappyHexMesh with a grid size of $12.5 \mu \mathrm{m}$. Grid independence of the initial droplet volume as well as the continuous flow profile with its converging maximum velocity in the membrane pore were checked. For this purpose, an adaptive mesh refinement at the droplet interface to a cell size of $3.125 \mu \mathrm{m}$ was applied to guarantee mesh independence of the applied surface tension via a continuum surface force model [16]. The solution was verified for $\Theta=90^{\circ}$ with a overall grid size of $10 \mu \mathrm{m}$ and a cell size at the interface of $2.5 \mu \mathrm{m}$. The solution may be mesh dependent, as the grid has to resolve the smallest droplet volume during break-up.

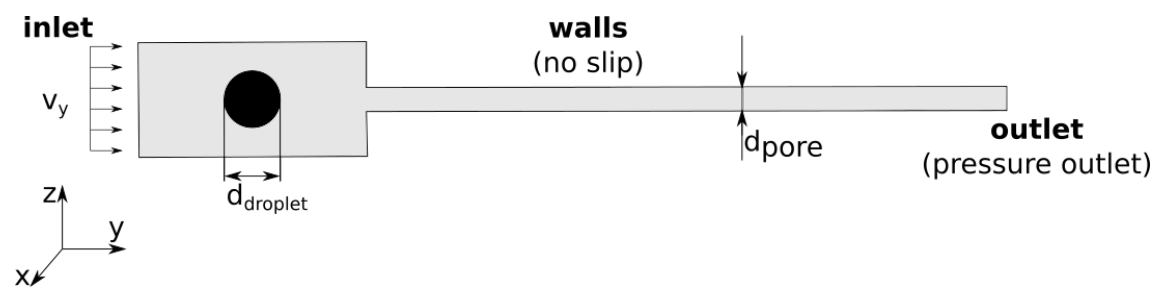

Figure 1. problem definition of idealized membrane pore (capillary tube)

Table 1. Simulation parameters

\begin{tabular}{cccc}
\hline Density $\frac{\mathrm{kg}}{\mathrm{m}^{3}}$ & Viscosity $\frac{\mathrm{m}^{2}}{\mathrm{~s}}$ & Interfacial Tension $\frac{N}{\mathrm{~m}}$ & Dimensions $\mu \mathrm{m}$ \\
\hline$\rho_{c}=1000$ & $\nu_{c}=1 e-6$ & $\sigma=0.024$ & $d_{\text {pore }}=200$ \\
$\rho_{d}=945$ & $\nu_{d}=2 e-5$ & & $d_{\text {droplet }}=500$
\end{tabular}

For all simulations presented in this paper, the time step $\Delta t$ is chosen to satisfy the Courant- 
Friedrichs-Lewy (CFL) criterion, for a chosen CFL number of 0.5 and time step restrictions to resolve capillary waves [16] as follows:

$$
\Delta t<\Delta t_{C L F}
$$

$$
\Delta t<\Delta t_{\sigma}=\sqrt{\frac{0.5\left(\rho_{c}+\rho_{d}\right) * \Delta x^{3}}{2 \pi \sigma}}
$$

With the grid spacing $\Delta x$.

\section{Results and discussion}

In the following section, we present the results and discuss the underlying breakup mechanisms. It is therefore divided into three main categories of the breakup mechanism: entrance in the pore, propagation and break-up. Membrane wettability is varied from $\Theta=0^{\circ}-180^{\circ}$ defined from the continuous phase. The results are shown for a Capillary Number of $C a=1.6$ and velocities are measured at a line shown in figure 2 in the entrance of the pore, where the first wall interaction takes place.

\section{Entrance in the pore}

For the entrance of the dispersed phase into the pore, two stages of interest are defined. As pointed out earlier, the pre-deformation of the droplet while entering is crucial and therefore analyzed. Furthermore, the first interaction of the dispersed phase with the solid membrane wall is investigated.

Due to the entrance of the pore and the related reduction of the flow cross-section, the droplet gets deformed. The velocity in the constriction is higher, the droplet volume inside the pore is accelerated and consequently elongated. The velocity profile inside the pore is shown in figure 3 for the stage where the droplet emerges from the pore as shown in 2(a) and (b) respectively.

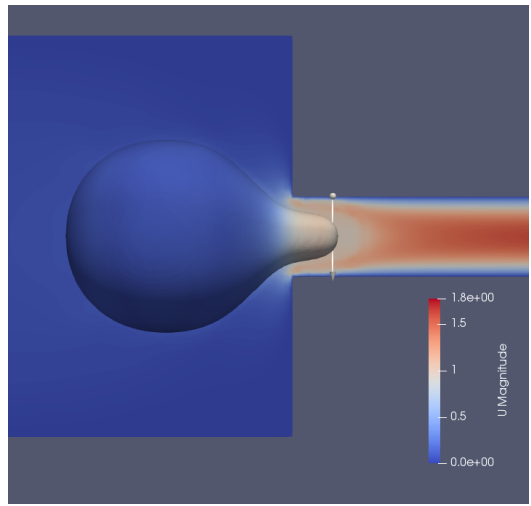

(a) droplet contour at $t=0.0146 \mathrm{~s}$

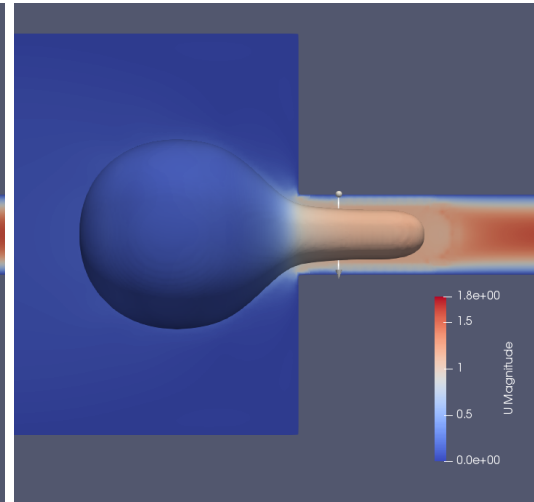

(b) initial deformation of droplet contour at $t=0.0150 \mathrm{~s}$

Figure 2. initial droplet deformation during entering the pore, white line at $y=0.000004 \mathrm{~m}$ indicates measurement line at for following figures

The profile shows velocity peaks in the vicinity of the wall for $t=0.0146 \mathrm{~s}$. This behavior can be explained by the higher velocity of the continuous phase flowing around the not fully accelerated dispersed phase. At this point the droplet is not interacting with the wall, consequently the velocity profile is independent of the wettability as shown in figure 3 . It can be noted that the velocity profile in the entrance is not fully developed as shown in figure 3(a). When the droplet 


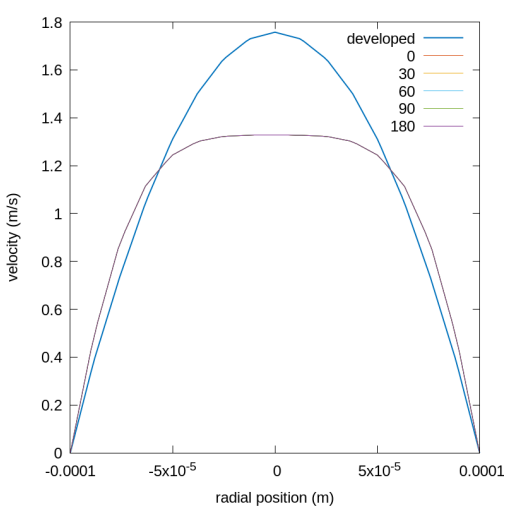

(a) $t=0.0142 \mathrm{~s}$

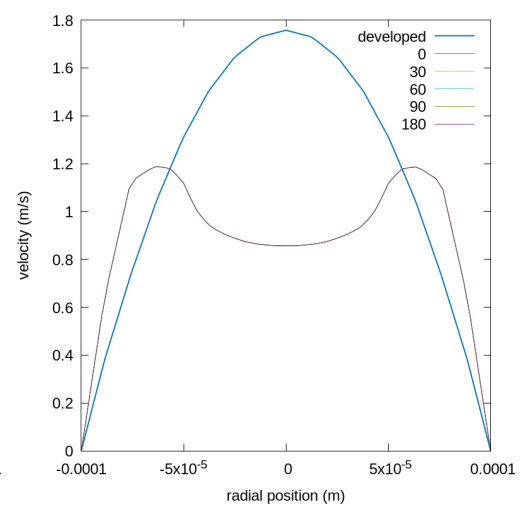

(b) $t=0.0146 \mathrm{~s}$

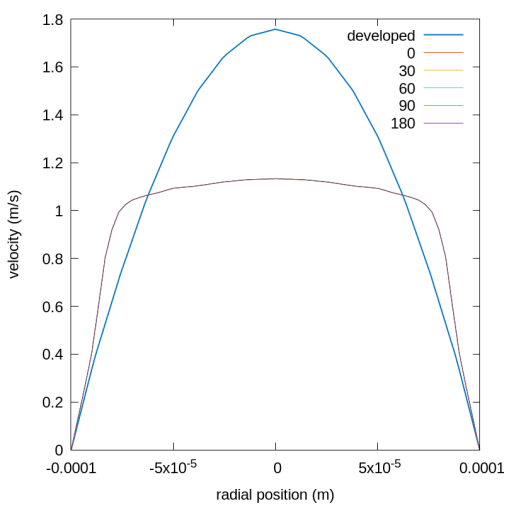

(c) $t=0.0150 \mathrm{~s}$

Figure 3. velocity profile at measurement line $y=0.000004 m$ before wall interaction for different times and wettability

further emerges into the pore, the velocity profile flattens as seen in figure 3(c) into a plug flow profile.

At $t=0.0154 \mathrm{~s}$ figure 4(c) shows the diameter of the emerging droplet at the constriction. A higher pre-deformation for the wetting system can be noted. With the first wall interaction, the wettability also affects the velocity profile as shown in figure 4(a)(b). The kinks in the velocity profile are connected to the remaining continuous phase in the vicinity of the wall and therefore due to fluid/fluid shear stress. The kink outside the Poiseuille profile is located at $\alpha=0.5$. The second kink on top of the fully developed profile is connected to shear stress of the viscous under layer in the entrance of the pore. Figure 4(b) shows the dependency of the wettability on the velocity component perpendicular to the walls at the measurement line mentioned previously.

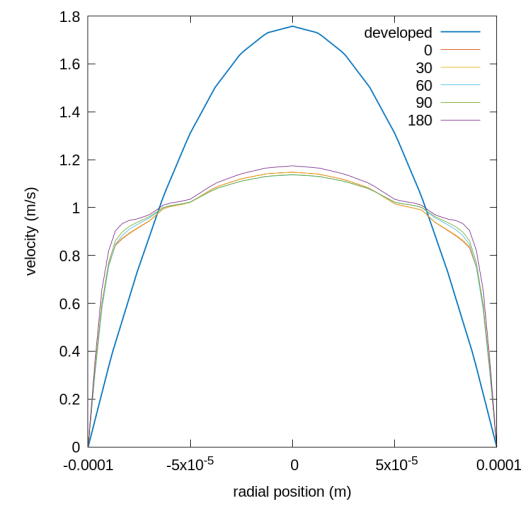

(a) velocity profile in $y$ direction at (b) measurement line $y=0.000004 m$

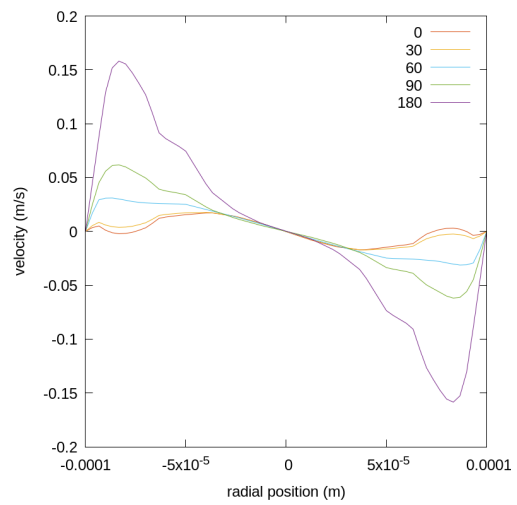

(b) velocity in $z$ direction measurement line $y=0.000004 m$

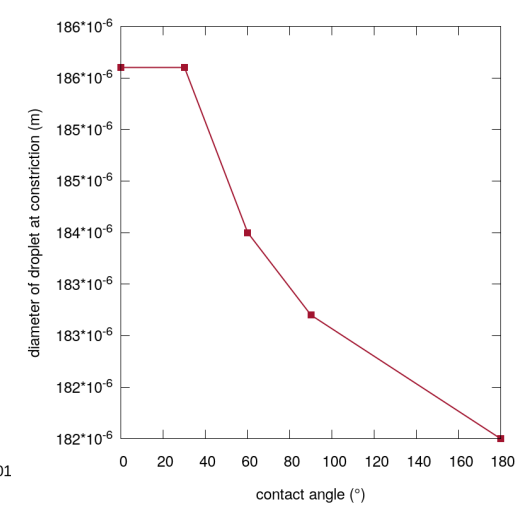

(c) droplet diameter at constriction $y=0$ for different wetting conditions

Figure 4. Velocity and necking of the droplet in the entrance of the capillary for $t=0.0154 \mathrm{~s}$ and varying wettability

\section{Propagation}

From figure 6 it is noted, that the center line velocity increases with increasing wettability. Furthermore, the slice through the dispersed phase shows a necking of the droplet for $\Theta>30^{\circ}$ as presented in figure 5 . The amplitude of the resultant interfacial perturbation shows dependency on the applied contact angle, as shown in figure 6(c). It is measured from the wall to the trough of the perturbation at $\alpha=0.5$ as shown in figure 5 (c). This contact line instability is formed by the advancing contact line in strong dependence on the interface mobility. It results in fingering 


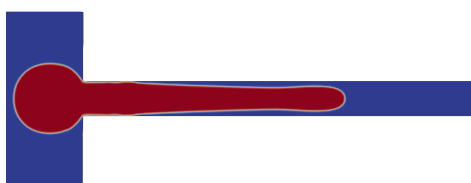

(a) interfacial perturbation for $\Theta=30^{\circ}$

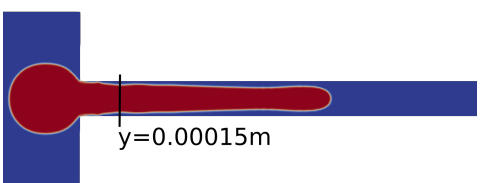

(b) interfacial perturbation for $\Theta=90^{\circ}$

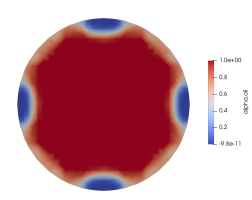

(c) radial slice through interfacial perturbation for $\Theta=90^{\circ}$ at $y=0.00015 m$

Figure 5. Interfacial perturbation at $t=0.0158 \mathrm{~s}$

instabilities as described by Thoroddsen et al. [17] The radial slice through the pore at the contact line instability shows the symmetrical but three-dimensional perturbation. It propagates in radial and axial directions.

As the droplet further emerges into the pore, the velocity profile approaches the Poiseuille profile shown in figure 6 . It is not fully reached as the kinks in the profile remain and the maximum center line velocity is lower. The velocity component perpendicular to the wall at this point does not differ significantly between the different wetting conditions (data not shown). At this point, it is interesting to note, that the laminar profile of a single phase flow at this measurement line is not fully developed. The resultant profiles are between the undeveloped and fully developed laminar profile. The velocity profiles for $t=0.0168$ mainly differ in their maximum velocity between different wetting conditions at this point of the dispersion.

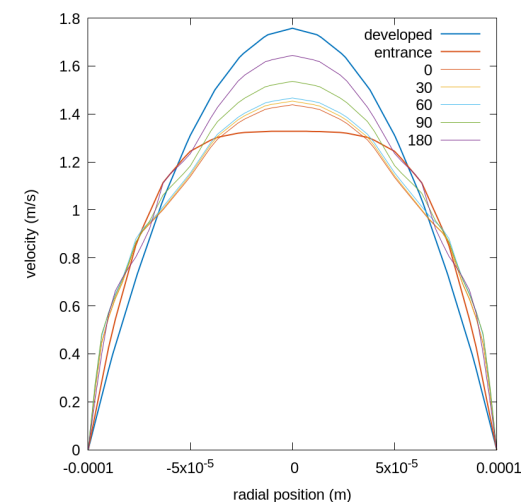

(a) $t=0.0158 \mathrm{~s}$

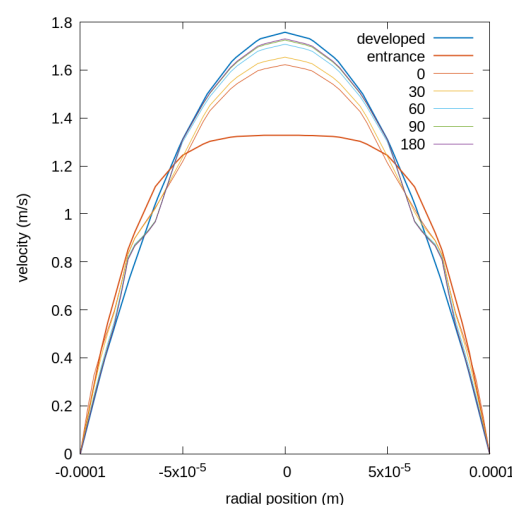

(b) $t=0.0168 \mathrm{~s}$

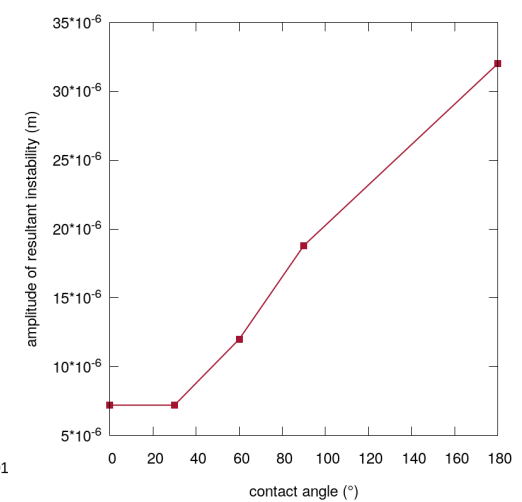

(c) amplitude of resultant instability at $t=0.0158 \mathrm{~s}$

Figure 6. velocity profile at pore entrance with wall interaction for different times and varying wettability

However, from figure 7 it is clear, that wettability has an effect on the pore entrance. In case of non- to partial-wetting, $\Theta=0^{\circ}-60^{\circ}$ the contact line recedes from the pore entrance and the remaining volume enters into the pore in a convex shape. In contrast, in the wetting-case $\Theta \geqq 90^{\circ}$ the contact line remains at the pore entrance and limits the dispersed phase volume to emerge into the pore, which results in a concave shape at the end of the entering droplet as the continuous phase is penetrating into the pore and the emerged droplet volume. Consequently, a backside deformation in strong relation to the dispersed phase in vicinity of the wall is formed and is therefore dependent on the contact angle. The center line velocity accelerates over the maximum value of the Poiseuille flow, as the dispersed phase narrows the flow cross-section and the instability propagates downstream. 


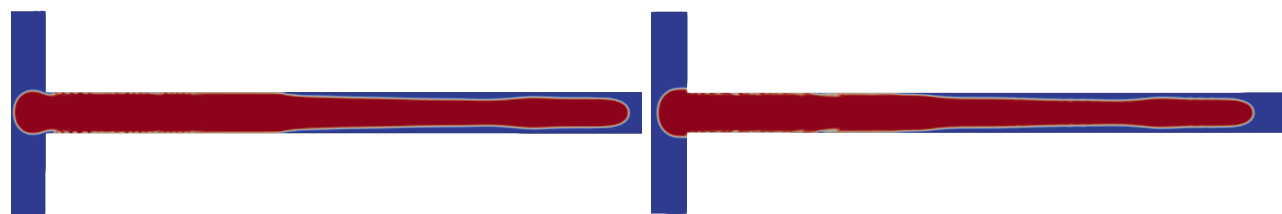
(a) $\Theta=30^{\circ}$
(b) $\Theta=60^{\circ}$

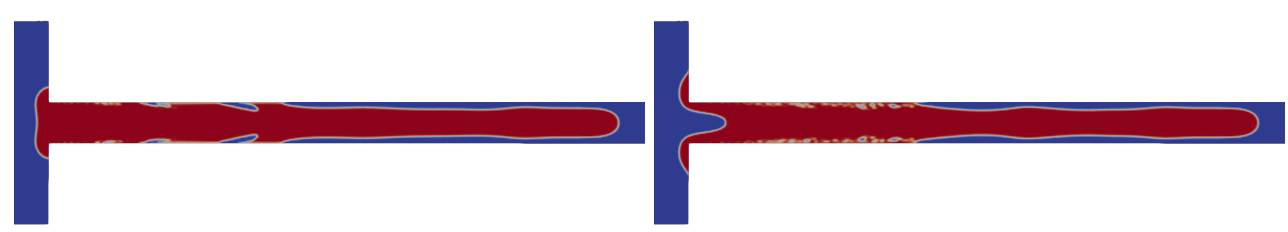
(c) $\Theta=90^{\circ}$
(d) $\Theta=180^{\circ}$

Figure 7. Interfacial perturbation at $t=0.017 \mathrm{~s}$

\section{Break-up}

With the initial contact line instability still necking the droplet for $\Theta>30^{\circ}$ the droplet breaks up when the backside deformation meets the contact line instability. As this backside deformation propagates further, it breaks up the droplet for all applied contact angles at the backside of the Rayleigh Plateau instability. This is the moment of the highest fluid/fluid shear stress. It has to be noted that there are several breakup events for $\Theta>30^{\circ}$ but in case of $\Theta>60^{\circ}$ the dispersed phase sticks to the membrane wall making the first break up ineffective. Additionally, for $\Theta \geqq 90^{\circ}$ phase inversion emulsification as described by Suzuki [1] can be observed at the membrane wall.

\section{Conclusions}

Different wetting conditions for a capillary driven droplet breakup have been numerically investigated. The results give insight into the driving mechanisms of droplet break up in capillary tubes. A strong relation between wetting properties and fluid dynamic disturbances' resp. instabilities which yield to droplet breakup are shown. The present study emphasizes the importance of wettability in this region of breakup, where no junction or cross-flow breaks the droplet apart. It gains further understanding on the dispersion improvement caused by surfactant and their implied effect on wetting properties. Especially the entrance area in particular and the associated undeveloped flow profile is of great importance. The higher velocity in vicinity of the wall of the undeveloped profile supports the transport of the dispersed phase towards the walls. The findings provide an explanation why membrane emulsification is particularly suited for shear sensitive media, as fluid dynamic instabilities play a major role in droplet breakup and reduce the shear stress threshold necessary for droplet breakup. Rayleigh instabilities are inversely proportional to the surface tension and can consequently not be the only driving force behind the breakup in such cases. In addition, for hydrophobic and super-hydrophobic surface modifications, a phase inversion was observed.

\section{Acknowledgements}

This project is embedded into the SPP 1934 "DiSPBiotech" funded by the German Research Foundation (DFG) and was supported by the North-German Supercomputing Alliance (HLRN). 


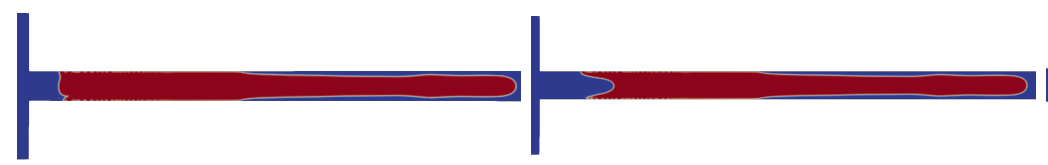
(a) $\Theta=30^{\circ}, t=0.0174 \mathrm{~s}$
(b) $\Theta=30^{\circ}, t=0.0176 \mathrm{~s}$
(c) $\Theta=30^{\circ}, t=0.0182 \mathrm{~s}$

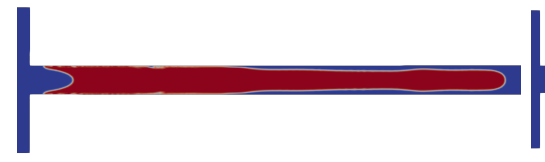

(d) $\Theta=60^{\circ}, t=0.0174 \mathrm{~s}$

(e) $\Theta=60^{\circ}, t=0.0176 \mathrm{~s}$

(f) $\Theta=60^{\circ}, t=0.0182 \mathrm{~s}$

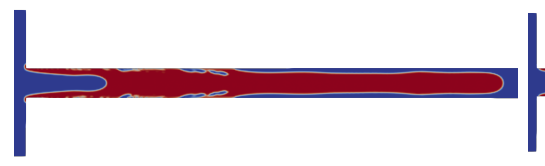

(g) $\Theta=90^{\circ}, t=0.0174 \mathrm{~s}$

(h) $\Theta=90^{\circ}, t=0.0176 \mathrm{~s}$

(i) $\Theta=90^{\circ}, t=0.0182 \mathrm{~s}$

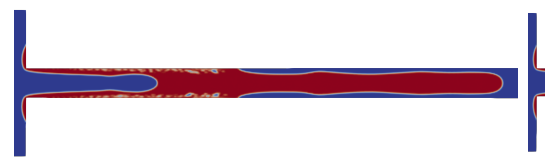

(j) $\Theta=180^{\circ}, t=0.0174 \mathrm{~s}$

(k) $\Theta=180^{\circ}, t=0.0176 \mathrm{~s}$

(I) $\Theta=180^{\circ}, t=0.0182 \mathrm{~s}$

Figure 8. Droplet breakup at initial interfacial perturbations for different wetting conditions

\section{Nomenclature}

$\alpha \quad$ phase fraction

$\Theta \quad$ Contact angle [o]

$\nu_{c} \quad$ Viscosity of continuous phase $\left[\frac{m^{2}}{s}\right]$

$\nu_{d} \quad$ Viscosity of dispersed phase $\left[\frac{m^{2}}{s}\right]$

$\rho_{c} \quad$ Density of continuous phase $\left[\frac{\mathrm{kg}}{\left(\mathrm{m}^{3}\right.}\right]$

$\rho_{d} \quad$ Density of disperse phase $\left[\frac{\mathrm{kg}}{\mathrm{m}^{3}}\right]$

$\sigma \quad$ Interfacial tension $\left[\frac{N}{m}\right]$

\section{References}

[1] Kanichi Suzuki, Koji Hayakawa, and Yoshio Hagura. "Preparation of High Concentration $\mathrm{O} / \mathrm{W}$ and W/O Emulsions by the Membrane Phase Inversion Emulsification Using PTFE Membranes." In: Food Science and Technology Research 5.2 (1999), pp. 234-238. DOI: $10.3136 /$ fstr.5.234. 
[2] Jun Wu et al. "Preparation of W/O emulsions by membrane emulsification with a mullite ceramic membrane". In: Desalination 193.1-3 (May 2006), pp. 381-386. DOI: 10 .1016/ j. desal.2005.08.026.

[3] Isao Kobayashi et al. "Microscopic observation of emulsion droplet formation from a polycarbonate membrane". In: Colloids and Surfaces A: Physicochemical and Engineering Aspects 207.1-3 (July 2002), pp. 185-196. DOI: 10.1016/s0927-7757 (02) 00093-6.

[4] Shinzo Omi. "Preparation of monodisperse microspheres using the Shirasu porous glass emulsification technique". In: Colloids and Surfaces A: Physicochemical and Engineering Aspects 109 (Apr. 1996), pp. 97-107. DOI: 10.1016/0927-7757 (95) 03477-3.

[5] Kazuhiko Kandori, Kazuko Kishi, and Tatsuo Ishikawa. "Formation mechanisms of monodispersed W/O emulsions by SPG filter emulsification method". In: Colloids and Surfaces 61 (Dec. 1991), pp. 269-279. DOI: 10.1016/0166-6622(91)80315-f.

[6] Zheyu Liu et al. "Emulsification in a microfluidic flow-focusing device: Effect of the dispersed phase viscosity". In: Fuel 283 (Jan. 2021), p. 119229. DOI: 10 . 1016/ j . fuel . 2020.119229.

[7] Gerald Muschiolik et al. "Protein-Stabilized Emulsions Prepared by the Micro-Porous Glass Method". In: Food Colloids. Elsevier, 2004, pp. 393-400. DOI: 10.1533/9781845698263. 6.393.

[8] Jean-Christophe Baret. "Surfactants in droplet-based microfluidics". In: Lab Chip 12.3 (2012), pp. 422-433. DOI: 10.1039/c1lc20582j.

[9] C Charcosset, I Limayem, and H Fessi. "The membrane emulsification process-a review". In: Journal of Chemical Technology \& Biotechnology 79.3 (Feb. 2004), pp. 209218. DOI: $10.1002 /$ jctb. 969.

[10] Harold P. Grace†. "Dispersion phenomena in high viscosity immiscible fluid systems and application of static mixers as dispersion device in such systems". In: Chemical Engineering Communications 14.3-6 (Mar. 1982), pp. 225-277. DOI: 10.1080/00986448208911047.

[11] Tobias Wollborn, Laura Luhede, and Udo Fritsching. "Evaluating interfacial shear and strain stress during droplet deformation in micro-pores". In: Physics of Fluids 31.1 (Jan. 2019), p. 012109. DOI: 10.1063/1.5064858.

[12] Karin Schroën et al. "Linking Findings in Microfluidics to Membrane Emulsification Process Design: The Importance of Wettability and Component Interactions with Interfaces". In: Membranes 6.2 (May 2016), p. 26. DOI: 10.3390/membranes6020026.

[13] S.J. Peng and R.A. Williams. "Controlled Production of Emulsions Using a Crossflow Membrane". In: Chemical Engineering Research and Design 76.8 (Nov. 1998), pp. 894901. DOI: $10.1205 / 026387698525694$.

[14] A. J. Abrahamse et al. "Process fundamentals of membrane emulsification: Simulation with CFD”. In: AIChE Journal 47.6 (June 2001), pp. 1285-1291. DOI: 10 . 1002 / aic . 690470606.

[15] Eduard van der Zwan et al. "Visualization of droplet break-up in pre-mix membrane emulsification using microfluidic devices". In: Colloids and Surfaces A: Physicochemical and Engineering Aspects 277.1-3 (Apr. 2006), pp. 223-229. DOI: 10.1016/j . colsurfa . 2005. 11.064 .

[16] J.U Brackbill, D.B Kothe, and C Zemach. "A continuum method for modeling surface tension". In: Journal of Computational Physics 100.2 (June 1992), pp. 335-354. DOI: 10.1016/0021-9991 (92) 90240-y.

[17] S. T. Thoroddsen and J. Sakakibara. "Evolution of the fingering pattern of an impacting drop". In: Physics of Fluids 10.6 (June 1998), pp. 1359-1374. DOI: 10.1063/1.869661. 